\title{
Home Use of a Wireless Intracortical Brain- Computer Interface by Individuals With Tetraplegia
}

\author{
John D. Simeral*, Senior Member, IEEE, Thomas Hosman, Jad Saab, Sharlene N. Flesher, Marco \\ Vilela, Brian Franco, Jessica Kelemen, David M. Brandman, John G. Ciancibello, Paymon G. Rezaii, \\ David M. Rosler, Krishna V. Shenoy ${ }^{\S}$, Senior Member, IEEE, Jaimie M. Henderson`, Arto V. \\ Nurmikko, Fellow, IEEE, and Leigh R. Hochberg
}

\begin{abstract}
Individuals with neurological disease or injury such as amyotrophic lateral sclerosis, spinal cord injury or stroke may become tetraplegic, unable to speak or even locked-in. For people with these conditions, current assistive technologies are often ineffective. Brain-computer interfaces are being developed to enhance independence and restore communication in the absence of physical movement. Over the past decade, individuals with tetraplegia have achieved rapid on-screen typing and point-andclick control of tablet apps using intracortical brain-computer interfaces (iBCIs) that decode intended arm and hand movements from neural signals recorded by implanted microelectrode arrays. However, cables used to convey neural signals from the brain tether participants to amplifiers and decoding computers and require expert oversight during use, severely limiting when and where iBCIs could be available for use. Here, we demonstrate the first human use of a wireless broadband iBCI. Based on a prototype system previously used in pre-clinical research, we replaced the external cables of a 192-electrode $\mathrm{iBCI}$ with wireless transmitters and achieved high-resolution recording and decoding of broadband field potentials and spiking activity from people with paralysis. Two participants in an ongoing pilot clinical trial performed on-screen item selection tasks to assess iBCI-enabled cursor control. Communication bitrates were equivalent between cabled and wireless configurations. Participants also used the wireless $\mathrm{iBCI}$ to control a standard commercial tablet computer to
\end{abstract}

This work was supported in part by NIH-NINDS UH2NS095548; the Office of Research and Development, Rehab. R\&D Service, Department of Veterans Affairs (N9228C, N2864C, A2295R, B6453R, P1155R); NIH-NIDCD R01DC009899 and R01DC014034; NIH-NBIB R01EB007401; The Executive Committee on Research (ECOR) of Massachusetts General Hospital; MGHDeane Institute; DARPA REPAIR; Wu Tsai Neurosciences Institute at Stanford; Larry and Pamela Garlick; Samuel and Betsy Reeves; The Howard Hughes Medical Institute; Conquer Paralysis Now 004698.

*J. D. Simeral is with the Center for Neurorestoration and Neurotechnology, Rehabilitation R\&D Service, Department of Veterans Affairs Medical Center, Providence, RI 02908 USA (CfNN) and with the School of Engineering and the Robert J. \& Nancy D. Carney Institute for Brain Science, Brown University, Providence, RI (e-mail: john_simeral@brown.edu).

T. Hosman is with CfNN and the School of Engineering, Brown University.

J. Saab was with CfNN and the School of Engineering, Brown University. He is now with Insight Data Science, Toronto, Canada.

S. N. Flesher is with the Department of Electrical Engineering and Department of Neurosurgery, Stanford University.

M. Vilela was with the School of Engineering, Brown University.

B. Franco was with the Department of Neurology, Massachusetts General Hospital, Boston, MA. He is now with NeuroPace Inc., Mountain View, CA.

J. Kelemen is with the Department of Neurology, Massachusetts General Hospital, Boston

D. Brandman was with the School of Engineering, Brown University. He is now with the Department of Surgery (Neurosurgery), Dalhousie University, Halifax, NS, Canada. browse the web and use several mobile applications. Within-day comparison of cabled and wireless interfaces evaluated bit error rate, packet loss, and the recovery of spike rates and spike waveforms from the recorded neural signals. In a representative use case, the wireless system recorded intracortical signals from two arrays in one participant continuously through a 24-hour period at home. Wireless multi-electrode recording of broadband neural signals over extended periods introduces a valuable tool for human neuroscience research and is an important step toward practical deployment of iBCI technology for independent use by individuals with paralysis. On-demand access to highperformance iBCI technology in the home promises to enhance independence and restore communication and mobility for individuals with severe motor impairment.

Index Terms-Brain-computer interface, clinical trial, motor cortex, neural engineering, wireless transmitter

\section{INTRODUCTION}

$\mathrm{N}^{\mathrm{s}}$ EUROLOGICAL disease or injury such as amyotrophic lateral sclerosis, stroke and cervical spinal cord injury can result in tetraplegia, loss of speech or locked-in syndrome. Brain-computer interfaces (BCIs) are being developed to

J. G. Ciancibello was with the School of Engineering, Brown University. He is now with the Center for Bioelectronic Medicine at the Feinstein Institute for Medical Research, Manhasset, NY.

P. G. Rezaii is with the Department of Neurosurgery, Stanford University.

D. M. Rosler is with the CfNN and the School of Engineering and the Robert J. \& Nancy D. Carney Institute for Brain Science, Brown University, and also with the Department of Neurology, Massachusetts General Hospital.

K. V. Shenoy is with the Departments of Electrical Engineering, Bioengineering and Neurobiology, Wu Tsai Neurosciences Institute, and the Neurosciences and Bio-X Programs, Stanford, and also with the Howard Hughes Medical Institute, Stanford University.

J. M. Henderson is with the Department of Neurosurgery and Wu Tsai Neurosciences Institute, Stanford University.

A. V. Nurmikko is with the School of Engineering, Department of Physics, and the Robert J. \& Nancy D. Carney Institute for Brain Science, Brown University.

L. R. Hochberg is with the Center for Neurorestoration and Neurotechnology, Rehabilitation R\&D Service, Providence VA Medical Center, and the School of Engineering and the Robert J. \& Nancy D. Carney Institute for Brain Science, Brown University, and the Center for Neurotechnology and Neurorecovery, Department of Neurology, Massachusetts General Hospital, Harvard Medical School.

${ }^{\S} \mathrm{K}$. V. Shenoy and J. M. Henderson contributed equally to this work. 
restore communication and motor function for individuals living with profound motor disability. Motor BCIs aim to provide access to assistive devices by decoding user commands from electroencephalography (EEG), electrocorticography $(\mathrm{ECoG})$, or intracortical signals. In ongoing clinical research, high-performance intracortical BCIs (iBCIs) are being developed to infer a user's movement intentions [1]-[8] or speech [9], [10] from neural activity recorded from one or more microelectrode arrays implanted in motor areas of cortex. By imagining natural hand, finger and arm movements, trial participants with paralysis have achieved reach-and grasp with robotic and prosthetic limbs [2], [3], [11] and their own reanimated limb [6], [12], and have demonstrated reliable cursor control for tablet use [8] and on-screen typing for communication [4], [7], [13]. Building on steady advances in point-and-click accuracy, speed [4], [7], [13]-[16] and consistency [17]-[21], iBCI trial participants at home have achieved average on-screen point-to-select typing rates over 37 correct characters per minute maintained over days and weeks [4], [7].

While this progress is promising, one practical limitation of current $\mathrm{iBCIs}$ is their reliance on recording cables that link an implanted array's head-mounted titanium connector ("pedestal") to the signal processing and decoding computers. Enabling iBCIs for long-term recording and independent mobile use at home without technical supervision will require wireless acquisition of intracortical signals to eliminate tethering cables to the head. However, wireless recording for iBCI has yet to be demonstrated in people, in part because translating the proven cabled system (16 bits per sample at 30 $\mathrm{kS} / \mathrm{s}$ for each of 96 electrodes per implanted array) to wireless form presents significant engineering challenges. Although wireless broadband signal acquisition might not be a design requirement for some targeted $\mathrm{BCC}$ applications [22], it allows for investigation of novel iBCI algorithms spanning the full spectrum of neural activity while supporting a wide range of fundamental electrophysiological research during untethered use. With this motivation and the goal of enabling continuous, independent use of an iBCI, previous work from our team created a compact, power efficient neurosensor that digitizes and wirelessly transmits broadband cortical activity from a 96channel chronically implanted microelectrode array [23]-[26]. A battery-powered pedestal-mounted form of that transmitter was demonstrated in animals during open-loop tasks and free home-cage behavior [23], [24]. A fully implanted inductivelycharged version was developed and tested in-vivo in non-human primates [25], [26] and is on a translational path for human use. Both the external and fully implanted devices were designed with a relatively high sample rate $(20 \mathrm{kS} / \mathrm{s}$ per electrode, 12 bits/sample) to support both fundamental human neuroscience research and investigational signal processing and decoding methods for high-performance iBCI systems.

Here we report translation of a wireless broadband intracortical BCI system to human use and evaluate its performance during use at home by two participants in the BrainGate pilot clinical trial. The external transmitter underwent commercial manufacturing and preclinical safety testing in preparation for human investigational use. Transmission frequency was configurable such that neural activity from two 96-channel intracortical arrays could be recorded simultaneously without interference. Two transmitters and associated commercial receiver hardware were integrated into the iBCI real-time signal processing system. Both participants then used the BrainGate system to complete a series of cursor-based point-and-dwell assessment tasks [1], [7], [27] to quantify $\mathrm{iBCI}$ performance in cabled and wireless configurations. We demonstrate that the wireless signals could be decoded with sufficient quality and reliability to support spontaneous user-paced point-and-click use of a tablet computer as reported for previous participants using a cable connection [8]. To verify wireless function in one representative $\mathrm{iBCI}$ use scenario, one participant completed dual-array wireless recording over 24 hours of daily activity, rest and sleep. Finally, we examined the underlying signal quality and digital sample integrity in the wireless data streams relative to the cabled system in both benchtop and in-home evaluations.

\section{METHODS}

\section{A. Participants}

Study participants T5 and T10 were previously enrolled in the pilot clinical trial of the BrainGate Neural Interface System (http://www.clinicaltrials.gov/ct2/show/NCT00912041). At the time of this study, T5 was a 63 -year-old man with a C4 AIS-C spinal cord injury (SCI) resulting in tetraplegia. T10 was a 35year-old man with a C4 AIS-A SCI resulting in tetraplegia.

All activities, including use of the wireless devices, were permitted by the US Food and Drug Administration (Investigational Device Exemption \#G090003) and the Institutional Review Boards of Partners Healthcare/ Massachusetts General Hospital, Providence VA Medical Center, Stanford University and Brown University.

For each participant, intracortical neural activity was recorded from two 96-channel planar silicon microelectrode arrays (4 $\mathrm{mm} \times 4 \mathrm{~mm}, 1.5 \mathrm{~mm}$ electrode length, platinum tips; Blackrock Microsystems, Salt Lake City, UT) placed in the left (dominant) precentral gyrus, except participant T10 whose second array was placed in the middle frontal gyrus.

\section{B. Standard Cabled iBCI System}

The cabled BrainGate iBCI used commercial hardware and software (Blackrock Microsystems) to acquire and record neural signals. This system included a NeuroPort Patient Cable connecting each percutaneous head-mounted pedestal to a Front End Amplifier which applied a hardware filter $(0.3 \mathrm{~Hz}-7.5$ $\mathrm{kHz})$ and digitized signals on each of 96 microelectrodes (30 $\mathrm{kS} / \mathrm{s}, 16$ bits per sample). The continuous serial stream of digital samples was relayed over fiber optic cable to a Neural Signal Processor (NSP) where they were timestamped and sent out as UDP packets on a private local area network (LAN). These "raw" data were stored (without software filtering or downsampling) by Blackrock's Central Suite software for offline 


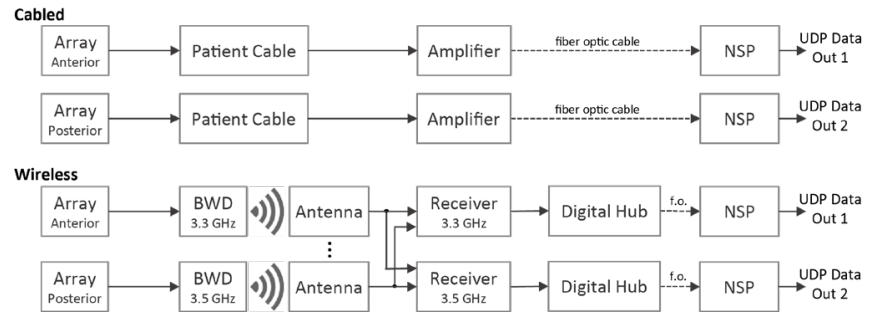

Fig. 1. Components of the cabled and wireless systems for dual-array recording. Pathways for neural signal acquisition differed as shown, but NSPs and all downstream file recording, signal processing and decoding hardware and software were the same for both systems.

analysis. Because participants in this study each had two arrays, the home iBCI included two parallel 96-channel acquisition systems (Fig. 1, top) that were time-locked by a sync cable linking the two NSPs.

The raw data packets were also delivered in real-time to downstream Assistive Technology computers for signal processing, neural feature extraction and decoding. The Assistive Technology system included a Super Logics x86 computer (Natick, MA) running the xPC Target real-time operating system (The Mathworks, Natick, MA) and custom signal processing and decoding algorithms running in Simulink. Decoded cursor commands were sent from Simulink to a Dell laptop (T5) or a Microsoft Surface tablet (T10) over wired Ethernet, wireless Ethernet (through a $5 \mathrm{GHz}$ WiFi router), or Bluetooth. Bluetooth communication employed our previously developed circuit board dongle based on BlueSMiRF technology (SparkFun Electronics, Boulder, CO) that plugged into the XPC Target computer and presented BrainGate decoder outputs as standard wireless mouse point and click commands [8], [28].

\section{Pedestal-Mounted Wireless Transmitter (BWD)}

The neurosensor deployed here (Fig. 2a, b) was designed from the outset for translation to human intracortical recording and in-home BCI applications. Design criteria included a high digital sampling rate to capture neuronal spiking activity, data acquisition and transmission over at least 24 hours for use throughout the day, and lightweight mating to the percutaneous pedestal that is currently the only interface available for chronic intracortical microelectrode recording in people (research only). Details of the design and characterization of the wireless transmitter have been reported previously for external [23], [24] and fully implanted [25], [26] forms. The external device was licensed to Blackrock Microsystems who made minor design refinements and manufactured it as the "Brown Wireless Device" (BWD). The BWD applies a hardware filter (1 Hz to $7.8 \mathrm{kHz})$, digitizes neural activity $(20 \mathrm{kS} / \mathrm{sec}, 12$ bits per sample) on each electrode, applies Manchester encoding and transmits it to nearby antennas using a custom low-power protocol. The Manchester encoding provides self-clocking, reduces link error rate and enhances reliability. In the digital data stream, a 24-bit "sync word" separates each $50 \mu$ s "frame" of data containing one 12-bit sample from each electrode. BWDs use a non-rechargeable medical-grade battery (SAFT LS14250, 3.6 V, $1000 \mathrm{mAh}$ ). Pre-clinical device safety assessments were completed prior to use in the BrainGate trial.

\section{Wireless iBCI System}

In the wireless recording system (Fig. 1, bottom), each NeuroPort Patient Cable and Front End Amplifier was replaced by four components (Blackrock Microsystems): a BWD, one or more polarized planar antennas $(5$ ” x 5 ", $3 \mathrm{GHz}-4 \mathrm{GHz}$ reception, PA-333810-NF), a Wireless Receiver (PN9323) and a Digital Hub (PN6973). The transmitter was designed as a lowpower, short-range (meters) broadband device operating in a low-use portion of the microwave communications spectrum. Each BWD digitized neural activity from one array and transmitted it at $3.3 \mathrm{GHz}$ or $3.5 \mathrm{GHz}$ (configured at time of manufacture) to the antennas. The corresponding receiver was manually tuned to the appropriate frequency. Each Digital Hub relayed the digital data stream to its respective NSP over fiber optic cable. The NSP, file recording system, and downstream Assistive Technology hardware and software were unchanged between the wired and wireless configurations.

The Wireless Receiver supported up to 8 antenna inputs in a Switched Diversity Single-Input Multiple Output (SIMO) architecture for improved reception. SIMO provides robust recovery of a single communication link (one BWD's continuous transmission) by allowing multiple antennas (e.g., in a home environment) where the signal can be retrieved (i.e., multiple outputs of the communication link). The receiver considered a data frame to be valid on any antenna input when the expected terminating sync word was detected ("signal lock") which was indicated by a blue LED on the front panel. The sample data in each frame did not include error checking, so it was possible for bit errors to occur in the data even when the sync word was detected. SIMO was used to provide a degree of data checking: when two or more receiver channels reported lock, the data output by the receiver was taken from a channel whose contents exactly matched those of another locked channel.

After preliminary wireless reception testing in the home of T10, we elected to reduce the physical footprint of the wireless system by using four (rather than 8 ) antennas mounted above and/or behind the user, 1-2 $\mathrm{m}$ from the pedestal-mounted transmitters. A tripod stand behind his bed allowed four antennas to be positioned near the head and adjusted to achieve effective angle and polarization orientations (Fig. 2c). For T5, two antennas were positioned on tables near his wheelchair and two at the ceiling slightly behind the wheelchair. During setup for each recording session, we ensured that LEDs on each receiver consistently indicated signal "lock" for each antenna input but did not attempt to further optimize the placement of antennas. As with the wired system, dual-array wireless recording required two parallel sets of equipment, except that the SMA cable from each antenna was split and connected to both wireless receivers. This avoided the need for duplicate antennas at the cost of splitting the signal power between the receivers.

To establish compatibility with the downstream data recording system used for this study, the wireless hardware (Wireless Receiver / Digital Hub) upsampled the BWD data stream from $20 \mathrm{kS} / \mathrm{s}$ to $30 \mathrm{kS} / \mathrm{s}$ (via sample-and-hold) and from 


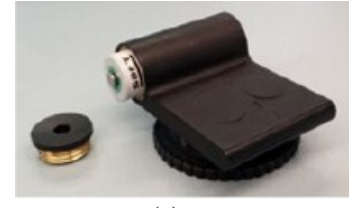

(a)

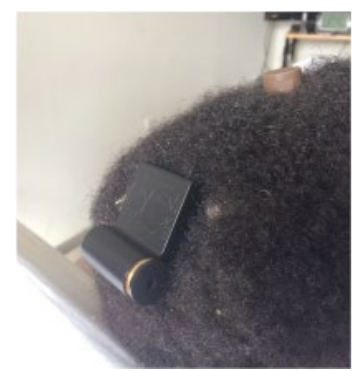

(b)

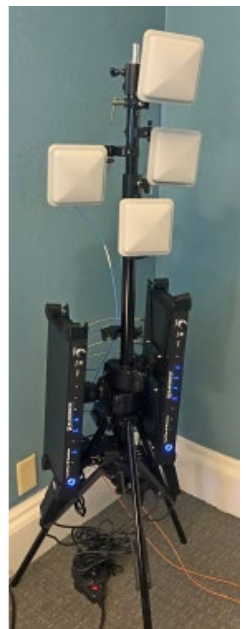

(c)
Fig. 2. Some components of the wireless system. (a) BWD transmitter $(5.2 \mathrm{~mm} \times 4.4 \mathrm{~mm})$ showing battery compartment. Turn-screw disc is used to attach the device onto a percutaneous pedestal. (b) The BWD connected to T10's posterior pedestal (the anterior pedestal is covered by a protective cap). (c) A two-frequency wireless receiver system in a four-antenna SIMO configuration as deployed for T10. The output optical fibers (orange) connect to downstream NSPs.

12 bits per sample to 16 bits per sample (four-bit up-shift). The upsampled wireless data was then stored in the standard ns5 file format and processed through the identical signal processing software as the cabled data. We applied a variety of analyses to assess the effect of these sample-level transformations on recorded neural signal content and $\mathrm{BCI}$ decoder function.

\section{E. Overview of Participant Research Sessions}

Prior to the first study session with T10, we performed an inhome spectral sweep for potential radio frequency (RF) interference with a microwave spectrum analyzer (Anritsu). No detectable sources of interference in the $3.0-4.0 \mathrm{GHz}$ band used by the BWD were found, despite numerous in-home appliances, medical devices, and a nearby line-of-sight commercial radio tower.

Participant sessions in this study included T10 trial days (post-implant day) 307, 349, 350, 355, 361 and T5 trial days $560,572,588$. Research sessions took place in participants' homes with oversight by each site's trained clinical neurotechnology research assistant (CNRA). T5 completed study activities seated in a wheelchair in his living room. For health reasons unrelated to the neural interface system, T10 completed all sessions while reclined in bed. Each participant completed two sessions in which cursor kinematics were controlled through decoded neural signals recorded in both the wired and wireless configurations. Each participant also completed a third session in which they used decoded pointand-click cursor control to navigate consumer software and web applications on a tablet computer. Sessions lasted approximately 3 hours, interrupted as needed by the participant's regular nursing and personal care activities. T10 also completed an overnight recording session spanning 24 continuous hours.

\section{F. Neural Decoding Methods}

For iBCI cursor tasks, a Kalman filter decoder and a linear discriminant classifier were used to estimate continuous 2-D cursor velocity and click state, respectively. After upsampling in the wireless path (described above), data processing and decoding algorithms were identical for cabled and wireless conditions as described next. The signal processing and decoding algorithms used here have been described in detail previously [4]. Briefly, signals were downsampled to $15 \mathrm{kS} / \mathrm{s}$ followed by common average referencing. For decoding, two neural features were then computed for each electrode: spike rates and spike power. Spike rates were computed from thresholded spikes. Spike power was estimated as the power in the high-frequency local field potential (HF-LFP). For both features, a band pass filter was first applied $(250 \mathrm{~Hz}-5 \mathrm{kHz}$, 8th order IIR Butterworth). Spike rates were then computed by refiltering the band-passed data in reverse to provide net zerophase shift [29] before applying a spike threshold at -3.5 standard deviations (computed for each channel from prior within-day blocks of data). Spike power was computed by squaring and averaging the band-passed signal. Spike power and thresholded spike rate features were z-score normalized using each feature's mean value and standard deviation calculated from a previous data block. All features, velocity, and state estimates were updated every $20 \mathrm{~ms}$.

\section{G. Decoded Cursor Control in Cabled versus Wireless iBCI}

During participant sessions, cabled versus wireless cursor performance was evaluated using an $\mathrm{A} / \mathrm{B}$ block design in which decoder calibration and several back-to-back Grid Task cursor assessments were completed in the cabled configuration (A) followed by the same sequence using the wireless system (B). For each condition, participants first completed a 3-minute long cursor-based closed-loop calibration task [27] which automatically computed coefficients of a 2-dimensional Kalman filter. For tablet control sessions, a discrete "click" state classifier was also calibrated. Participants commanded cursor movement and dwell using attempted hand (T10) or finger (T5) movements. Clicks were commanded using attempted hand grasp (T10) or attempted thumb tap (T5). After decoder calibration, several back-to-back Grid Task assessments were completed [1], [7], [27] to quantify iBCIenabled closed-loop point-and-select performance. Switching between cabled and wireless configurations involved powering down the iBCI equipment, removing the NeuroPort Patient Cables, connecting BWDs to the two pedestals, replacing the Front End Amplifier fiber-optic cable into the NSP with the fiber optic cable originating at the Digital Hub, and restarting the hardware and software. Decoder coefficients were randomly re-initiated prior to the automatic calibration step that started each series. In another series on the same or different day, the entire sequence was reversed to achieve a B/A block design (wireless first) to balance possible order effects.

\section{H. Grid Task Assessment of iBCI Cursor Control}

Decoder performance in wired and wireless conditions was 
assessed on a series of Grid Tasks ( 2 minutes each) in which participants moved the cursor to the indicated target and then dwelled on the target to select it. The Grid Task consisted of a $6 \times 6$ grid of adjacent squares in which all points were selectable as described previously [1], [7], [30]. Dwelling on any target for 1 second selected it (resulting in a correct or error selection) after which the next target was shown. Selections continued uninterrupted until the Grid Task ended. If no target was selected within 8 seconds then a timeout resulted in an error trial and the next target was presented. The Grid Task was repeated several times with brief pauses in between. Task performance was assessed for each 2-minute task in terms of Percent Correct target acquisitions (hit rate) and Bitrate quantifying information transfer (bits per second) as reported previously for this task [31]. Metrics of cursor control included Trial Duration (average time to acquire targets including successful dwell time and the timeout duration for error trials, in seconds), Path Efficiency (ratio of actual trajectory length from starting point to the target relative to the straight line path), and Angle Error (the mean angle between instantaneous trajectory and the direct-to-target trajectory, in degrees).

\section{Wireless iBCI Use of a Tablet Computer}

To evaluate whether the wireless neural interface system could enable individuals with paralysis to achieve reliable point-and-click control of a computer in their homes, T5 and T10 used the wireless iBCI for point-and-click control of an unmodified consumer mobile device (Microsoft Surface Pro tablet) for web browsing, use of consumer applications, and typing communication. Cursor control was enabled using our standard automated decoder calibration, then CNRAs placed the tablet comfortably in front of the participant and routed the iBCI decoded cursor and click commands to the tablet [8]. Participants used the tablets in Microsoft Windows 10 "tablet" mode to navigate the desktop and to start and use different programs of interest to them. Additionally, T5 was asked to complete several blocks of typing using Windows Notepad to provide estimates of typing performance. Although the native onscreen keyboard offered word prediction, T5 avoided these and selected all letters, capitalization ("shift" function), spaces and punctuation explicitly. For each typing block we computed correct characters per minute (ccpm) as the number of appropriate character selections including spaces and punctuation (excluding characters resulting in misspelling, "shift" selected for capital letters, and delete or backspace key selections) and dividing by the elapsed time. Detailed analysis of application use and typing rates were performed through offline analysis of screen capture recordings.

\section{J. 24-Hour Wireless Recording}

T10 completed an overnight in-home study to collect nearly continuous intracortical data over a 24 -hour period. The study began with a typical CNRA-administered BrainGate research session in which data were collected during point-and-click cursor calibration and performance assessment. A similar research epoch was completed the next day to end the 24-hour period. Between these administered research periods, the wireless system was configured to continuously record and store raw neural data for offline analysis. For this first-ever overnight recording, two CNRAs maintained continuous monitoring on a rotating schedule. During this time, T10 remained in bed for reasons independent of this study, but he engaged in his typical daily activities such as eating, watching television, conversing by phone, direct family interactions, and sleeping. He received his regular nursing care including rotations in bed every few hours. Because RF pathway obstructions were anticipated as caregivers stood or moved between the transmitter and antennas, and because body rotations could alter orientation of the two transmitters relative to each antenna, a second pole of 4 antennas was added for this overnight study (increasing the number of antennas from 4 to 8). Based on laboratory testing, the battery in each transmitter was proactively replaced every 10 hours to avoid any possibility of unanticipated signal loss due to battery drain. (The wireless transmitter was designed for 48 hours of continuous use per battery [23]; after this study, a correction to the BWD restored it to $48+$ hours). A video camera captured snapshots of the room once per minute (with participant permission) to document activity. File recording software (Cerebus Central File Storage App, Blackrock Microsystems) automatically split neural data into contiguous files (with no sample loss) to limit the size of individual files.

Offline, integrity of the recorded data was analyzed in 5minute segments contiguously across the 24-hour period (288 segments for each array). For visualization, the spectral content was computed from $0.5 \mathrm{~Hz}$ to $100 \mathrm{~Hz}$ in each segment. A "disrupted" segment was one with missing recorded data (e.g., brief stops between CNRA sessions and overnight recording), dropped packets, or pervasive noise (identified as segments in which the summed spectral power from $40 \mathrm{~Hz}$ to $100 \mathrm{~Hz}$ exceeded the $90^{\text {th }}$ percentile of the entire 24 hour period).

\section{K. Comparing Cabled and Wireless Signal Fidelity}

We conducted a series of $\mathrm{A} / \mathrm{B}$ experiments to more directly compare the fidelity of broadband recordings using the standard 96-channel NeuroPort Patient Cable "reference system" and the 96-channel wireless system. We compared noise, bandpass filtered signals, sorted spike waveforms and unit firing rates. These tests were performed in the lab and with participants at home.

Bench Measurements. An initial baseline comparison of signal fidelity was performed using a single NeuroPort Patient Cable or BWD transmitter to record well-defined signals from a Neural Signal Simulator (NSS, Blackrock Microsystems). Four antennas were placed within three meters and direct lineof-sight of the wireless transmitter to reduce the possibility of wireless data drops that could confound these baseline evaluations. The neural signal simulator provided continuous, well-defined patterns of voltages simulating slow local field potential oscillations $(1 \mathrm{~Hz}, 3 \mathrm{~Hz}, 9 \mathrm{~Hz}$ and $20 \mathrm{~Hz})$, three different repeating single-unit action potential waveforms on each channel, and baseline noise. Several blocks of broadband data ( $\sim 5$ minutes each) were recorded in consecutive wired and 
wireless configurations and then analyzed offline. Because the signal simulator generated deterministic spiking patterns, it was possible to precisely align and compare the continuous raw recorded signals and spikes (waveforms) across conditions. To objectively compare spiking units without operator bias [32], we first applied automated spike-sorting to each data set using a previously reported algorithm [33] and compared the resulting sorted unit waveform shapes, spike rates, and SNR measures.

Intracortical Signals. These head-to-head comparisons evaluated data recorded with $\mathrm{T} 5$ and T10 during the closed-loop point-and-click Grid Tasks recorded in multiple interleaved cabled (A) and wireless (B) data blocks within each assessment day. A/B studies were repeated in a different order on separate days with both participants. As with bench measurements, comparisons of recorded spiking activity were made after automated spike sorting.

To compare signal amplitudes across wired and wireless conditions, we applied an empirical gain adjustment to match absolute voltages in the recorded signals. Gain stages in the commercial wireless hardware were designed to achieve the same theoretical total gain as the wired hardware. However, the precise cumulative gain in each recording system is sensitive to normal variability in device fabrication parameters. These resulted in a small but measurable and consistent gain difference between wired and wireless recorded signals. We calibrated absolute voltages by finding the gain multiplier that minimized differences in the NSS simulated spike waveforms recorded in the wired and wireless bench tests. Based on these empirical measurements, we applied a $+2.97 \%$ amplitude adjustment to the wireless data for these comparisons.

\section{Digital Sample Integrity}

Transmission data loss is a concern for any wireless system. Here, wireless data loss occurred whenever the receiver did not find the expected sync word on any input channel. When this occurred, the current samples from all 96 electrodes were invalid and the previous valid frame was inserted into the data stream. This frame error was detectable in the recorded data as a back-to-back repeat of the previous 96-channel data sample. We quantified the number of frame errors in the received 20 $\mathrm{kS} / \mathrm{s}$ wireless data by examining the $30 \mathrm{kS} / \mathrm{s}$ data recordings and accounting for the expected back-to-back sample repeats from the upsampling process. The practical effect of wireless data loss depends not just on the number frame errors but on their distribution. Individual dropped frames (one $50 \mu$ sample from each electrode) distributed sparsely throughout the data stream should be less detrimental to $\mathrm{BCCI}$ decoding than contiguous data loss [22]. We quantified the prevalence of longer intervals of data loss using the standard Severely Errored Seconds (SES) metric that reports the number of non-overlapping 1-second periods in which the proportion of data errors exceeds a predetermined threshold. Here, a commonly used threshold of $50 \%$ found all 1 -second periods in which frame errors corrupted at least $500 \mathrm{~ms}$ of the data.

We interrogated the wired and wireless $30 \mathrm{kS} / \mathrm{s}$ recorded data for all instances of voltage changes exceeding a pre-determined biologically plausible slew rate ( $500 \mu \mathrm{V}$ per $33 \mu$ s sample). These included bit-flips within single samples resulting in a large voltage excursion that returned to baseline in the following sample (or next sample after that in the case of upsample repeats), and large amplitude noise events extending over many samples before returning to baseline. We examined the degree to which each event was isolated to individual channels or observed simultaneously across many electrodes of the recording array.

\section{RESULTS}

\section{A. Translation of the Wireless Sensor for Human Studies}

The commercialized BWD underwent pre-clinical device testing in accordance with U.S. Food and Drug Administration (FDA) Good Laboratory Practice (GLP) guidelines. BWD testing verified safe levels of charge transfer; reliable sterilization (ANSI/AAM/ISO 10993-7-2008); immunity to repeated direct and indirect electrostatic discharge (ESD) up to $+/-6 \mathrm{kV}$ and $+/-8 \mathrm{kV}$, respectively (IEC 61000-4-2). Radio frequency (RF) transmission power measured well under 1 $\mathrm{mW}$, below the specific absorption rate (SAR) exclusion threshold of $16 \mathrm{~mW}$ (FCC 447498 D01 General RF Exposure Guidance). The transmitter was operated in high density RF environments ranging from a major hospital to a metropolitan city center with no interference from such telecommunicationsrich environments. The FCC granted an Experimental License to operate the BWD in the $3.3 \mathrm{GHz}-3.8 \mathrm{GHz}$ transmission band in geographical regions related to the BrainGate trial.

\section{B. Assessment of Wireless Point and Click Cursor Control}

We evaluated if the cabled and wireless $\mathrm{BCI}$ configurations enabled equivalent kinematic decoding and closed-loop cursor performance by BrainGate trial participants in their homes. On each of two days, participants successfully achieved cursor control using an automated calibration procedure which generated the Kalman decoder coefficients used in the subsequent Grid Task assessment blocks. Across two sessions, T10 completed 172 trials over 6 blocks in the cabled configuration and 167 trials over 6 blocks in the wireless configuration. Participant T5 completed two sessions as well totaling 922 trials over 20 blocks in the cabled configuration and 1,023 trials over 24 blocks in the wireless configuration.

The percentage of successful target selection was not significantly different between wired and wireless systems (Wilcoxon rank sum $>0.05$ ) for either participant (Fig 3a). T5 median success rate was $96.2 \%$ versus $97.9 \%$ in the cabled and wireless conditions, respectively. T10 achieved $91.6 \%$ and $94.8 \%$ in cabled and wireless configurations. Bitrates in the Grid Tasks were also not significantly different (Fig. 3b) between cabled and wireless for either participant (Wilcoxon rank sum > 0.05; T5: 1.58 bps cabled and 1.77 bps wireless; T10: 1.47 bps for both systems).

These statistics provided one measure per task iteration. We also quantified cursor control at the resolution of individual trials (Fig. 3c). Across three metrics (trial duration, path 


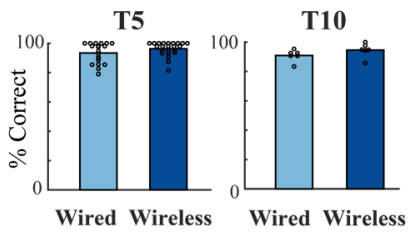

(a)

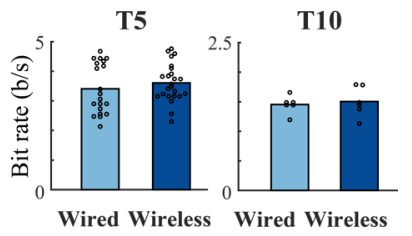

(b)
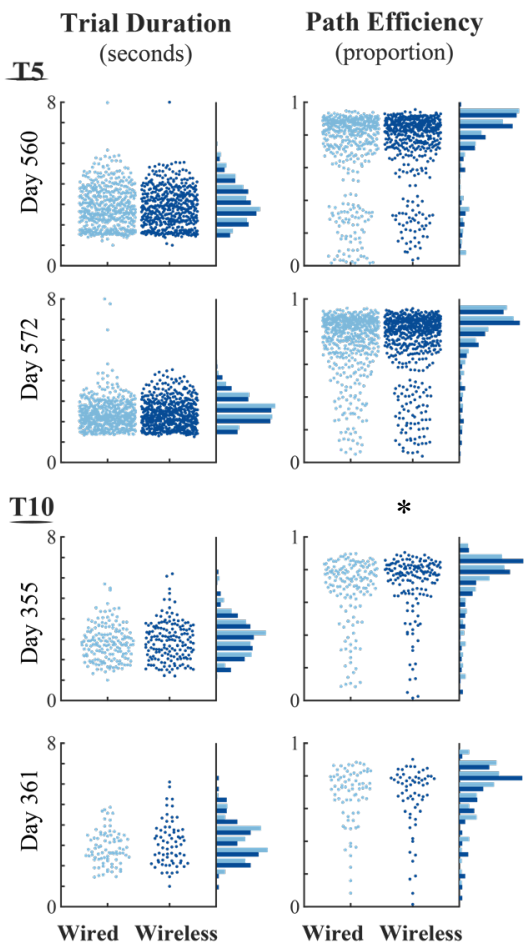

Wired Wireless
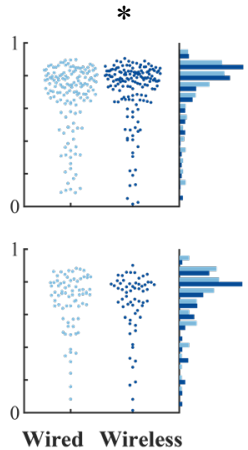

(c)
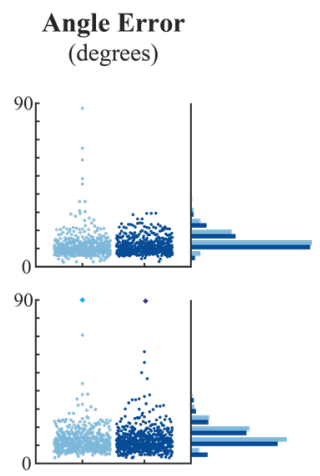

$*$
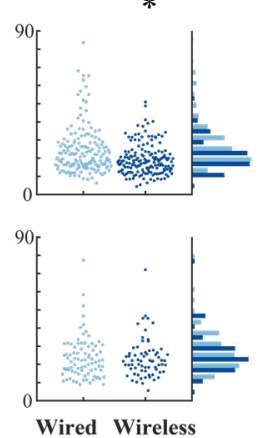

Wired Wireless
Fig. 3. Metrics comparing closed-loop cursor control in wired (light blue, left half of each figure) and wireless (dark blue) configurations. (a) Median target acquisition rates in wired and wireless conditions. Circles indicate the measure for each iteration of the Grid Task across two sessions for each participant. (b) Bitrates in wired and wireless conditions (one measure for each grid task across two sessions for each participant). (c) Three metrics of cursor control over two days for each participant. Each point shows the metric computed for an individual trial (one target acquisition). Points are spread on each $\mathrm{X}$-axis to reveal individual trials. Histograms on the right of each plot summarize wired and wireless performance.

efficiency, angle error), no significant differences were observed on either day with T5 or on trial day 361 with T10. Significant differences were observed only for T10 path efficiency and angle error on day 355 (Wilcoxon rank sum < $0.05)$. On this day, the median measures for both path efficiency ( 0.74 wired, 0.78 wireless) and angle error (20.7 degrees wired, 18.0 degrees wireless) were better with the wireless system. While the causes of this slight apparent superiority of the wireless system are not clear, the wired condition exhibited more outlier trials with low path efficiency and high angle error on this day.

\section{Wireless iBCI Use of a Tablet Computer}

T5 and T10 each used the wireless $\mathrm{BBCI}$ to achieve point-andclick control of a standard consumer Microsoft Surface 2-in-1 tablet computer running the Windows 10 operating system. On trial day 307 , after completing an autocalibration sequence and

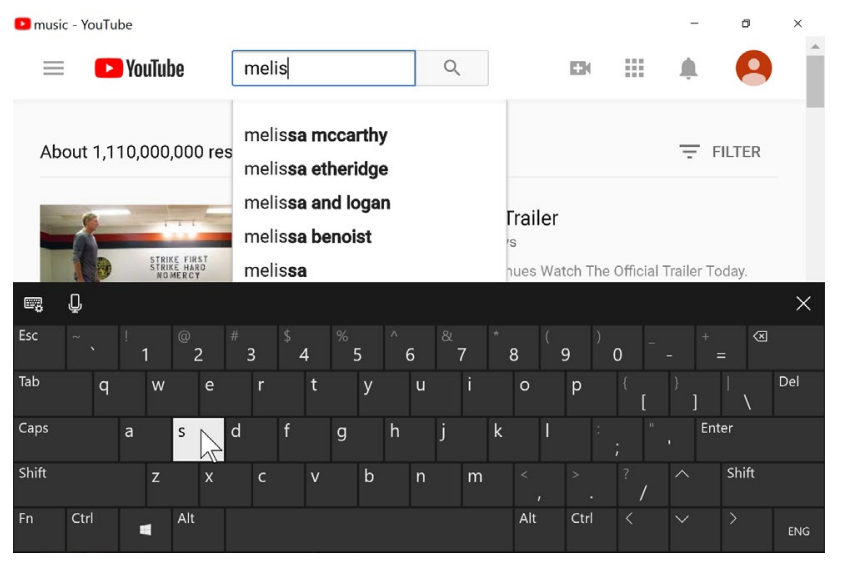

Fig 4. Screen capture of T5's tablet while he used the iBCI for point-andclick text entry with the built-in on-screen keyboard to search a web site for music by Melissa Etheridge.

several Grid Task assessment blocks, T10 started several widely-used apps in sequence by selecting the corresponding app tile on the Windows start menu or desktop. He used the Microsoft Edge browser, Pandora, the NCAA basketball app, Skype, YouTube, Gmail and the Weather app (Table 1). T5 used these same apps (excluding NCAA) on his trial day 588, including typing on the Windows on-screen keyboard to perform searches (Fig. 4). T5 also typed sufficient spontaneous text in the Windows Notepad to provide a basic measure of typing performance. Over 814 seconds (14.6 minutes), T5 typed 196 correct characters; any characters contributing to misspelling or that $\mathrm{T} 5$ subsequently deleted were included in the elapsed time but not in the character count.

Table 1. Tablet apps used by T5 during wireless point-and-click control.

\begin{tabular}{|ll|}
\hline \multicolumn{1}{|c|}{ App } & \multicolumn{1}{c|}{ Activity } \\
Pandora & $\begin{array}{l}\text { Click to start Pandora in a web browser, select an } \\
\text { artist, click the play button, select the "Thumbs } \\
\text { Up" icon, click Pause, return to desktop }\end{array}$ \\
Skype & $\begin{array}{l}\text { Select app tile on the desktop, select a game } \\
\text { "recap' icon, select the game video to play }\end{array}$ \\
YouTube & $\begin{array}{l}\text { Select the Skype icon in the start menu, select a } \\
\text { contact and start a voice call, talk for 90 s }\end{array}$ \\
& $\begin{array}{l}\text { Start app from the start menu, select on-screen } \\
\text { keyboard icon, type search term, select a video, } \\
\text { select "full-screen" icon, click back to desktop } \\
\text { Gmail }\end{array}$ \\
Click on Inbox icon, select a message and read it \\
Click through weather forecasts
\end{tabular}

\section{Wireless Acquisition During 24 Hours in the Home}

In T10's 24-hour study, synchronized neural data were wirelessly recorded two intracortical arrays from $2: 22 \mathrm{pm}$ trial day 349 to 2:22 pm trial day 350 . With pre- and post-session activities, total participant engagement time was 26 hours. Each array logged $500 \mathrm{~GB}$ of broadband data (1 TB for 2 arrays over 24 hours).

We examined the spectral content of the recorded signals for evidence of transmitted neural activity and/or recording artifacts. Spectral content of the record across the 24 hours was evaluated in contiguous 5 minute segments. Results for the 
posterior pedestal (recording from the intracortical array in the precentral gyrus) are shown in Figure 5. Prominent LFP activity was observed in bands centered at $10.6 \mathrm{~Hz}$ and $19.5 \mathrm{~Hz}$. Clear changes in the spectral power were evident during the night and early morning (e.g., in the $\sim 20-40 \mathrm{~Hz}$ band) corresponding to the period when T10 was observed to be sleeping (approximately 3 am to 9 am).

Over the 24 hours $(1,440 \mathrm{~min}$.), wireless recording was highly reliable. Wireless recording was uninterrupted throughout most of T10's activities, including using a head tracking system to type emails and browse the web, having a phone conversation, watching TV, talking, eating, sleeping and more. However, "disruptions" in the form of unrecorded data or noise were observed in 35 of the 288 five-minute analysis segments (175 total disruption minutes). A review of the session logs found that the large majority of data disruptions (100 minutes) occurred when one or more caregivers were attending to T11 including rotating or shifting him in bed, suctioning, and other nursing care (Table 2). During these periods, caregivers worked in close proximity to the bed including standing directly between the transmitters and antennas for several minutes at a time. Data were recorded but exhibited packet loss which was sometimes accompanied by substantial noise when the signal was recovered. In other cases, data flow stopped entirely when transmitters were removed during battery replacement, bathing and changing. Other disruptions were unrelated to the wireless components but rather resulted from technical glitches in the real-time data filing process. Only 15 minutes of data disruption were not readily associated with noted activities; these occurred during sleep between 6:45AM and 7:15 AM.

Table 2. Events associated with disrupted wireless signals over 24 hours.

\begin{tabular}{|cl|}
\hline Total Minutes & \multicolumn{1}{c|}{ Activity } \\
100 & Caregivers attending T11 (rotate in bed, suction) \\
25 & Bathing, dressing (transmitters removed) \\
15 & Unexplained signal noise during sleep \\
15 & Glitches in real-time file processing system \\
10 & Battery changes \\
10 & File breaks before/after cursor sessions \\
\hline
\end{tabular}

\section{E. Benchtop Comparison of Cabled and Wireless Recordings}

To provide a baseline comparison of the two systems, we performed benchtop tests in which well-defined simulated neural signals from the Neural Signal Simulator were recorded through the cabled and wireless pathways. The NSS generated continuous broadband data which superimposed noise, lowfrequency oscillations and "action potentials" mimicking three spiking neurons with distinct waveform shapes. After applying a spike-band filter and aligning corresponding simulated spike waveforms, there was a close correspondence between the signals recorded with the two systems (Fig. 6a). We observed low overall noise levels in both conditions with a few microvolts more noise (rms) in the spike band with the wireless system (Fig. 6b; wired noise: $11.2 \mu \mathrm{V}$; wireless: $13.5 \mu \mathrm{V}$ ) and

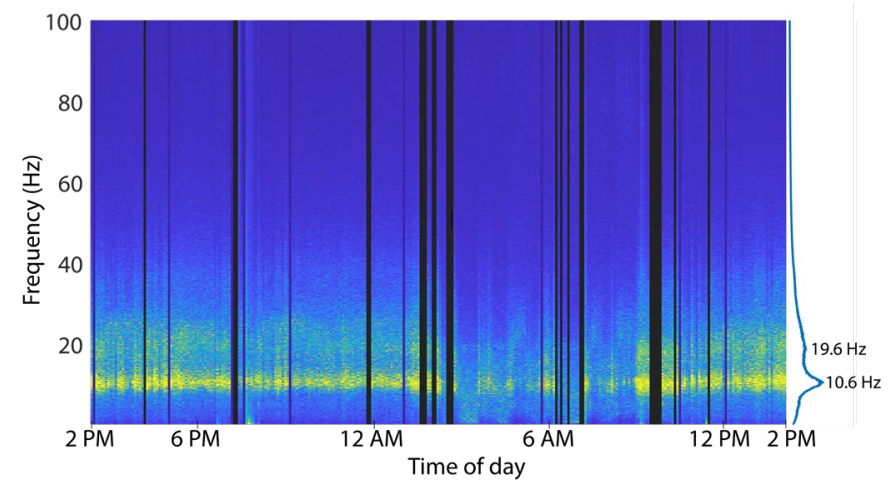

Fig 5. Spectral content of T10 neural data recorded continuously over 24 hours with the wireless system. X-axis indicates wall-clock time. Dark vertical bars reflect periods where data was not recorded (e.g., transmitters removed) or was severely disrupted (high frame loss).

$10 \mu \mathrm{V}$ more noise with the cable in the LFP-filtered band (Fig. 6c; wired: $166 \mu \mathrm{V}$; wireless: $156 \mu \mathrm{V}$ ).

To evaluate the fidelity of spike recording, we applied an offline automated unsupervised sorting algorithm to recover the waveform shapes and firing rates on all electrodes. The simulator's three unique waveform shapes were all reliably recovered on all electrodes as indicated by equivalent waveform sorting templates in wired and wireless conditions. The firing rates of all units on all 96 channels were identical $(3.6 \mathrm{~Hz})$ and matched the simulated rates, confirming that action potentials were recorded with the requisite fidelity. The equivalence of units recovered from wired and wireless data sets was evident when mean waveforms and their standard deviation were overlaid (Fig. 7).

\section{F. Wirelessly Recorded Human Intracortical Signals}

In a similar way, we compared human data recorded with wired and wireless systems during all Grid Tasks. The raw (unfiltered), LFP-filtered, and spike-filtered signals were substantially similar across conditions (Fig. 8a). Noise in the spike-filtered intracortical data was slightly lower than observed with the simulator. Consistent with the NSS tests, noise in the spiking band was a few microvolts higher in the wireless condition (Fig. 8b). Spike waveforms were equivalent between the recording modalities as demonstrated by overlaying wired and wireless waveforms for putative single units (Fig. 8c). We extracted waveforms for spiking units on all electrodes, applying the same unsupervised algorithm and parameters to wired and wireless data recorded during Grid Tasks. Comparable single unit waveforms were extracted across most electrodes. However, several units were observed in only one condition or the other. These were usually, but not always, small amplitude units or waveforms that were inconsistently split into multiple units. Thus, the population of extracted units was not identical between wired and wireless data. However, we also observed similar variability between Grid Task blocks recorded with the cable alone. Given the fidelity of recording confirmed in our simulator tests, these discrepancies were most plausibly attributed to nonstationarities in the recorded intracortical signals [17] rather than failures in the wired or wireless systems. 

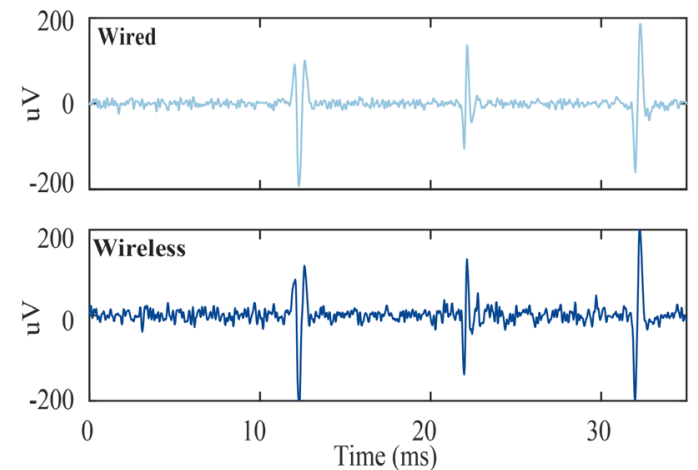

(a)

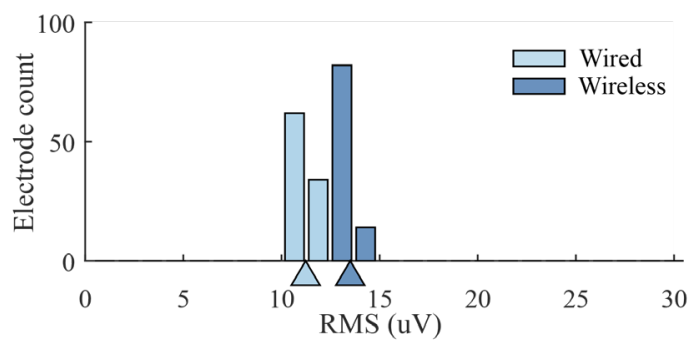

(b)

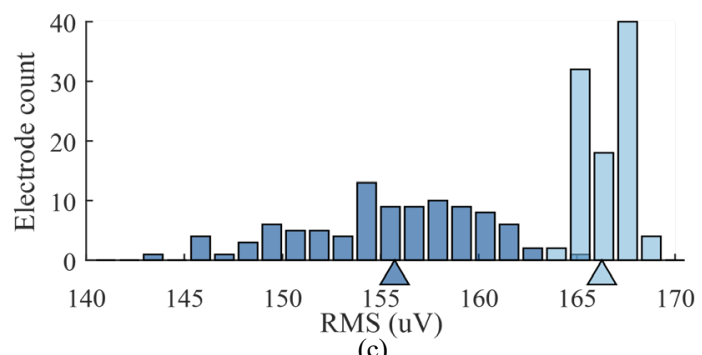

(c)

Fig. 6. Comparison of wired and wireless recordings of simulated neural signals. (a) Waveforms of three different simulated spiking neurons aligned from bandpass-filtered wired and wireless data. (b) Distribution of noise across 96 channels filtered for spiking data $(250 \mathrm{~Hz}-7.5 \mathrm{kHz})$; baseline noise was measured after spike events were removed by thresholding. Triangles indicate median rms values. (c) Distribution of noise across LFP bands $(1 \mathrm{~Hz}-250 \mathrm{~Hz})$. T10.2018.04.16 002(1)

\section{G. Wireless Packet Loss During In-Home iBCI Use}

To quantify the incidence of data loss during use of the wireless system, we inspected all raw $30 \mathrm{kS} / \mathrm{s}$ data recorded from each participant's two arrays during all Grid Tasks completed by T5 (24 blocks, 47.9 min) and T10 (6 blocks, 10.3 min) over 2 days each. A "packet drop" occurred when the sync word delimiting a $50 \mu$ s wireless data frame containing a single sample from all electrodes could not be recovered by the receiver, resulting in a repeat of all electrodes' previous digital values being re-inserted into the receiver's output stream.

The incidence of dropped packets varied across participants and pedestal position (Table 3). For each participant, signal recovery was consistently more successful for one transmitter (anterior $3.3 \mathrm{GHz}$ for $\mathrm{T} 5$, posterior $3.5 \mathrm{GHz}$ for $\mathrm{T} 10$ ) than the other. For T5, packet drops were observed during 8 of 24 blocks from the anterior pedestal and during all 24 task blocks from the posterior pedestal; the proportion of dropped packets was overall very low $(0.003 \%$ of all frames, anterior; $0.4163 \%$ of all frames, posterior). No packet drops were ever observed for

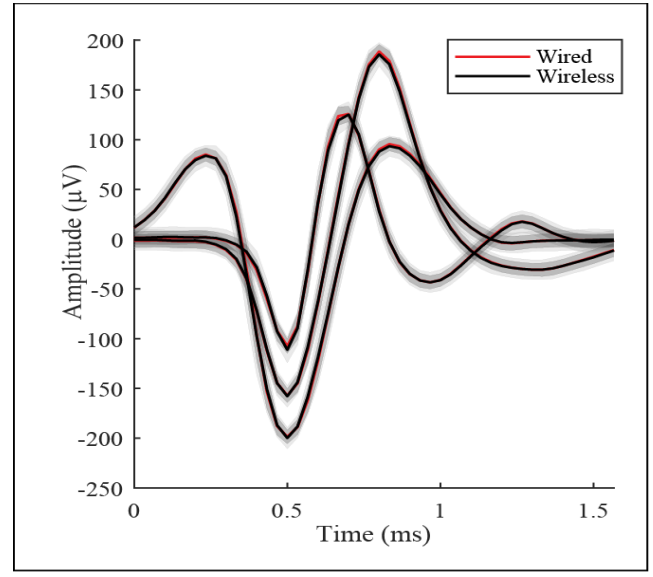

Fig. 7. Mean waveforms of the three simulated neurons as extracted from one representative electrode recorded by the cabled (red) and wireless (black) systems. Each unit shows mean (red or black line) +/- s.d. (gray bands) waveform averaged over a random selection of spikes.

T10's posterior pedestal and rarely for the anterior pedestal (fewer than 5 total packet drops per block). In one block, however, T10's anterior packet drops occurred with a drop rate of $4.69 \%$. We attribute differences in the fraction of dropped packets among blocks to changes in orientation of the participant (transmitters) relative to the antennas. Although participants had limited movement, the four antenna configurations that we used to simplified session setup in the home may not have provided sufficient simultaneous spatial coverage for both directions of transmission from two BWDs.

Table 3. Incidence of wireless data packet drops during Grid Tasks.

Data for simultaneous anterior (Ant) and posterior (Pos) data streams.

\begin{tabular}{|c|c|c|c|c|c|c|c|c|c|c|}
\hline ID & \begin{tabular}{|} 
\# Task \\
Blocks
\end{tabular} & $\begin{array}{c}\text { Total } \\
\text { Time } \\
\end{array}$ & \multicolumn{2}{|c|}{$\begin{array}{c}\text { \# Blocks with } \\
\text { Dropped Packets }\end{array}$} & \multicolumn{2}{c|}{$\begin{array}{c}\text { \% of All Data } \\
\text { Dropped }\end{array}$} & \multicolumn{2}{|c|}{$\begin{array}{c}\text { of Blocks } \\
\text { with SES }\end{array}$} & \multicolumn{2}{|c|}{$\begin{array}{c}\text { Total SES } \\
\text { Time (sec) }\end{array}$} \\
\hline T5 & 24 & 47.9 & 8 & 24 & 0.003 & 0.416 & 0 & 5 & 0 & 10 \\
\hline T10 & 6 & 10.3 & 6 & 0 & 1.004 & 0 & 0 & 0 & 0 & 0 \\
\hline
\end{tabular}

It was noteworthy that during the 2-minute block with high packet drop, T10 nonetheless acquired 44 of 45 targets and recorded his highest bit rate in this study $(1.791 \mathrm{bps})$. This could reflect the fact that, during this block, data from the second transmitter experienced 0 dropped packets. The performance impact of packet drops may also relate to whether they are sufficiently distributed to avoid extended periods of impoverished neural decoding. To quantify this, we computed the number of Severely Errored Seconds (SES), any nonoverlapping 1-second period in which half or more of the data samples were lost. Although packet drops occurred, SES events were rarely observed, limited to T5's posterior data stream in 5 blocks with a cumulative total of 10 seconds. During this study, a total of 6984 wireless data seconds (nearly 2 hours) were recorded during the Grid Task assessments cumulatively across both participants and both arrays; 10 of these exhibited SES.

\section{H. Bit Level Analysis of Wireless Data}

We examined the raw data files for any evidence of bit-level anomalies in the $30 \mathrm{kS} / \mathrm{s}$ data upsampled from each transmitter. These analyses excluded epochs with signal anomalies 

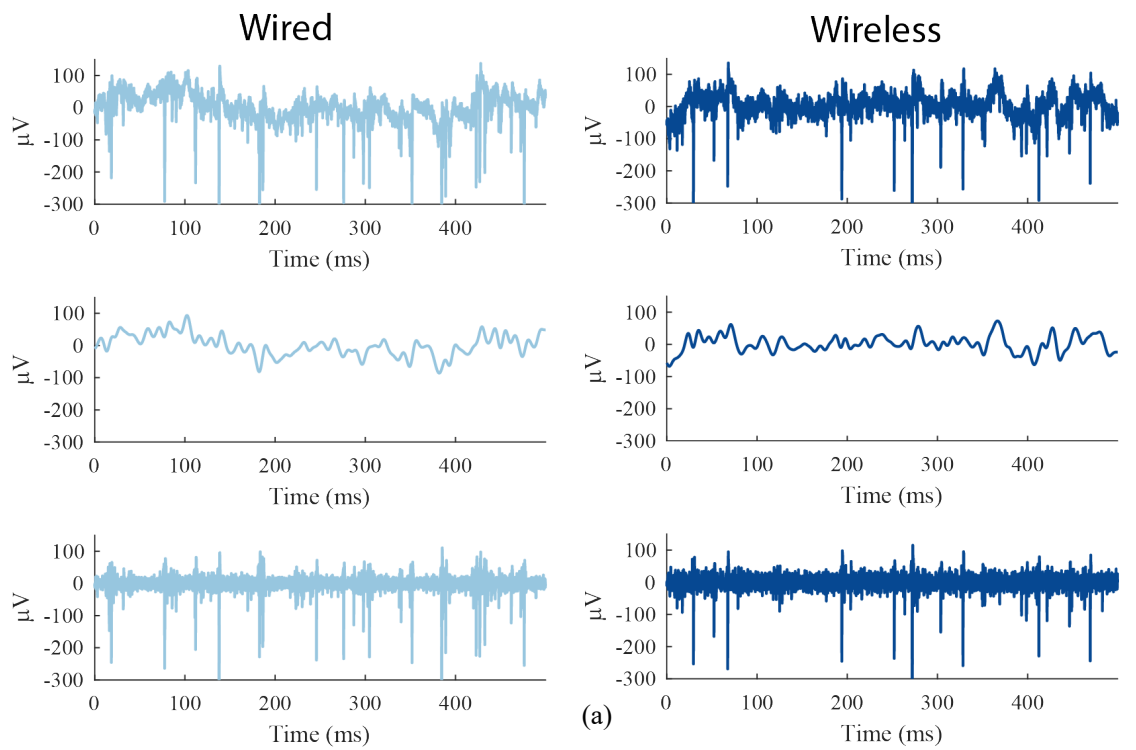

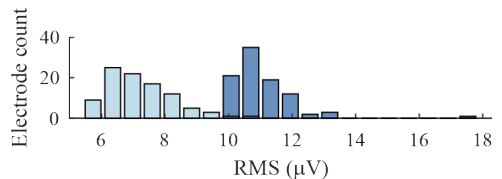

(b)

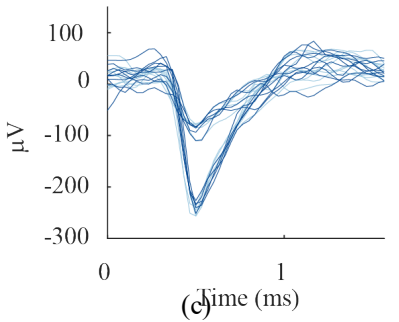

Fig. 8. Human intracortical signals recorded in wired and wireless configurations in the home. (a) Comparison of recorded neural activity on one electrode (t10.2017.06.05.e24 blocks 6, 7). Top: the "raw" unfiltered neural signal. Middle: low-pass filtered (100 Hz cutoff). Bottom: band pass filtered for spike extraction $(250 \mathrm{~Hz}-7.5 \mathrm{kHz})$. (b) Distribution of residual rms amplitudes from all electrodes on one array after bandpass filtering and removing thresholded spikes for wired (light blue) and wireless (dark blue) recordings. (c) Sample waveforms from two units sorted from the same electrode shown in (a) and (b). Light blue (wired) and dark blue (wireless) waveforms show substantial similarity.

attributed to dropped packets (analyzed above). The wireless data exhibited occasional instances of large instantaneous voltage discontinuities. These were biologically implausible voltage differences between consecutive $33 \mu$ s samples caused by bit flips among the most significant bits (MSBs) of a digital sample. The corrupt voltage value lasted only one sample (or two samples when an upsample repeat followed) then returned to baseline in a single step. These fast single-sample slew rates were sometimes observed among the lower bits as well, and it seems probable that lower bits were equally prone to flipping but the corresponding small voltage fluctuations would generally have been indistinguishable from baseline activity (and, presumably, functionally inconsequential). The incidence of MSB errors was inconsistent across channels and across blocks. In any given recording, some channels could be recorded error free while others exhibited rare, or more frequent, bit errors. Nonetheless, bit errors were observed in every wireless recording (at a rate of $2.04 \mathrm{E}-5$ to $9.26 \mathrm{E}-5$ per sample throughout the 30 Grid Task blocks). Another form of bit flip persisted for three or more samples and recurred multiple times over a period of tens or hundreds of milliseconds with seemingly valid sample data in between. These periods of recurring MSB errors, which we termed "digital noise", presented as noisy epochs in the voltage data. Digital noise events were rare in our Grid Task data but more likely in the 24-hour data. In general, we observed a higher incidence of all bit errors including digital noise after periods of packet drops and in association with putative antenna switching events. Bit errors also appeared to be associated with the upsampling logic (see below). Bit errors could have been introduced at various stages of the wireless system such as the BWD analog-to-digital converter, the wireless link, SIMO antenna switching, and the clock domain boundary in the upsampling process. The data collected here did not allow concrete identification of the source(s) of these errors. MSB errors were not observed in the cable-recorded data.

Because MSB errors were identified in our preliminary work, this study applied an algorithm to detect MSB-corrupted samples (briefly, any voltage shift $500 \mu \mathrm{V}$ or greater within a single sample, i.e., a $15 \mu \mathrm{V} / \mu$ s slew rate) and replace each with its preceding good sample on that electrode. In this way, the impact of flip-bit errors was minimized in real-time, prior to feature extraction and decoding, and in offline analyses.

As expected by design, the recorded wireless data contained a large proportion of $33 \mu$ s data frames that contained perfect copies of the prior samples on all 96 electrodes. These "upsample repeats" resulted directly from the sample-and-hold logic that upsampled the $20 \mathrm{kS} / \mathrm{s}$ BWD data to the NSP $30 \mathrm{kS} / \mathrm{s}$ format. Unexpectedly, one or more bits in the upsample repeat frame occasionally differed from their original value. Although these anomalies needed to be accounted for during analysis, they did not otherwise impact wireless performance.

\section{Discussion}

This study demonstrates the first human use of a broadband wireless intracortical brain-computer interface. Two human subjects directed computer cursor movements and click decoded by an iBCI that acquired and wirelessly transmitted (previously prototyped as [24]) broadband neural activity from 192 chronically implanted microelectrodes. Across multiple signal and $\mathrm{BBCI}$ performance metrics, the wireless solution proved to be a thorough replacement for the cabled connection currently used in chronic human iBCIs studies and fundamental non-human primate research. Raw neural data were processed into spike rates and LFP power in the spike band which were decoded together to yield precise $\mathrm{iBCI}$ cursor control. Using the 
wireless iBCI, study participants achieved communication bit rates in a Grid Task equivalent to the wired system. Self-paced cursor control enabled both participants to browse the web and complete other tablet activities, and one participant used the wireless interface for free typing. The wireless technology also enabled continuous recording of intracortical broadband field potentials and spiking activity from one participant over 24 hours in his home. Untethered recording of intracortical signals and in-home iBCI use are major steps toward an on-demand iBCI to provide independent digital communication and computer access for people with severe motor impairment.

Evaluation of the raw neural signals and spike waveforms found that signal processing differences in the cabled and wireless pathways had negligible impact on the quality of the recorded neural signals. A small increase in baseline noise with the wireless system was detectable but had no functional impact. The wireless system did exhibit bit errors which we were able to mitigate with real-time algorithms. It proved possible to record broadband intracortical signals in the home without packet drops, as demonstrated by many data blocks in which one or the other transmitter exhibited zero packet drops. Packet drops that did occur were exceedingly rare, indicating the fundamental reliability of the wireless system. Because wireless signal recovery is sensitive to plane of transmitter orientation and distance relative to the position and polarization orientation of the receiving antennas, we anticipate that packet drops could be reduced by more optimal placement of the antennas or by using the full complement of antennas supported by the receivers. Critically, the fidelity of the broadband neural signals and $\mathrm{BBCI}$ performance were robust to the low incidence of packet drops that was observed in most blocks. During 24hour in-home recording we found that volitional head movements and even body rotation by nursing staff generally did not interrupt the wireless signal. However, someone standing or working close to the participant's head could block transmission entirely, resulting in data loss or "digital noise" during some periods of nursing care. For this study, we elected not to modify users' homes or wheelchair to accommodate more robustly-placed or permanent antennas. However, these findings motivate further investigation of optimal antenna placement in the home.

\section{A. Design trade-offs}

The BWD system deployed in this study prioritized uncompromising signal fidelity and high electrode count to facilitate both iBCI applications and fundamental human electrophysiology research. However, as the field progresses toward higher and higher electrode counts, technologies for broadband recording become increasingly challenging and alternative, lower-bandwidth signal acquisition approaches are being considered. Design specifications for a wireless targeted BCI multielectrode acquisition system could be simplified by limiting signal acquisition to spiking events (with or without sampled spike waveforms) without broadband data. Although sorted single-unit activity and threshold crossings contain distinct information [34], [35], decoding thresholded multiunit activity rather than sorted units can enable precise iBCI control in monkeys [36]-[38] and in our own BrainGate participants with tetraplegia [4], [7]. Decoders built on multiunit thresholds can also be maintained over long periods [4], [7], [39] and population dynamics may be estimated from multiunit threshold crossings without spike sorting [40]. An analysis of iBCI decoding in monkeys and humans proposes that digital sampling at $1 \mathrm{kHz}$ should be sufficient for iBCI applications [22]. These approaches could reduce the wireless bandwidth by roughly an order of magnitude, thereby simplifying the engineering design constraints for a fully implanted wireless iBCI or, alternatively, freeing bandwidth to record from thousands of electrode contacts [41]-[46].

Despite the broadband design requirement, the BWD design also prioritized low power for long battery life to facilitate users with disability using the system throughout their day. The low power design also translates into fewer inductive charging periods for the fully-implanted version of the BWD (currently in pre-clinical testing). There are several clear trade-offs, however, and comparison to other systems is informative. A pedestal-mounted wireless interface with a commercial WiFi chipset has been used in an unconstrained non-human primate [47]. While similar to the BWD in terms of signal sampling and channel count, the package was relatively large, had high power consumption and incorporated a cooling fan. A subsequent commercial device modified from our prototype, Cereplex-W (Blackrock Microsystems) prioritized slightly lower noise, 16bit $30 \mathrm{kS} / \mathrm{s}$ sampling, error correction in the wireless link, and more powerful transmission - with the tradeoff of requiring much higher power consumption than the BWD and therefore much shorter operation per battery charge.

Other broadband wireless systems for multielectrode research have been demonstrated in freely moving primates [48]-[53] and a growing number of commercial alternatives support wireless intracortical recording for animal research. To our knowledge none are yet approved for human use, but several designs have a level of integration and other design tradeoffs that could be consistent with future translation. For any of these systems, engineering and translational challenges toward chronic human use include pairing the wireless components to intracranial electrodes suitable for long-term implantation in people, a small and lightweight package, high data rates (and/or on-board processing) across many electrodes, long run time (low power consumption), successful safety testing and completion of regulatory activities.

\section{B. Comparison to Other Wireless Implanted BCIs}

An early wireless broadband BCI demonstrated the ability for an ALS patient to toggle a binary signal from a single intracortical electrode [54]. One early ECoG closed-loop system with 32 subdural electrodes enabled an individual with tetraplegia [55] to move a cursor to targets by associating four different visualized movements with four cursor directions. Although neither wireless nor chronic, this system demonstrated moderate multidimensional cursor control based on decoded intracranial signals. A novel approach to intracranial recording, via electrodes attached to an endovascular stent [56], is now in clinical feasibility trials. Such 
systems, while very low bandwidth, may represent an effective solution for some individuals with severe disability.

Recently, deep brain stimulators (DBS) have served as a convenient, clinically accepted platform upon which fully implanted wireless BCIs have been developed [57]-[59]. A wireless BCI, configured from a bi-directional fully implanted DBS device (Activa $\mathrm{PC}+\mathrm{S}$, Medtronic, Inc.) was used to sample field potential data $(0.8 \mathrm{kS} / \mathrm{s})$ from a bipolar pair of chronic subdural ECoG electrodes in a person with ALS [58]. The wireless link from the subclavicular transmitter $(11.7 \mathrm{kbps})$ was more than $1,000 \mathrm{x}$ lower bandwidth than one BWD; nevertheless, this enabled the individual to use the BCI to make click selections in a commercial scanning letter interface. The research participant spelled 44 prompted words at a mean rate of 1.15 correct characters per minute (without letter prediction). Despite the slow communication rate, the participant reported satisfaction with the system. In another study, the $\mathrm{PC}+\mathrm{S}$ system was used for clinical stimulation and ECoG recording of motorrelated potentials from five patients with Parkinson's Disease over the span of a year [59]. Up to 9 minutes of data from two subdural sites were recorded at a time and then downloaded for offline analysis. Although no closed-loop study was attempted, changes in beta and gamma bands were observed during movement, possibly relevant to a future closed-loop BCI implementation. The W-HERBS 128-channel ECoG system with $1 \mathrm{kS} / \mathrm{s}$ sampling per channel has been developed over many years but not yet tested in-vivo [60]. An ECoG system specifically designed for translation, the WIMAGINE® ECoG neural interface (Clinatec, Grenoble, FR), was recently demonstrated in an individual with spinal cord injury [61]. Two devices were placed over bilateral motor cortical areas for chronic epidural recording. The wireless data link supported 586 samples/second $\mathrm{x} 32$ contacts using external antennas held near the implants by a custom helmet. Using the BCI to activate a neural on/off switch, the participant was able to initiate walking movements of an avatar and an exoskeleton. The subject also commanded continuous control of bilateral limb movements of the exoskeleton and was able to steer a powered wheelchair. We have not yet evaluated the current wireless system relative to intracortical cabled control of robotic, prosthetic, or functional electrical stimulation applications [1][3], [11], [12]. However, the higher electrode count and 100x data rates demonstrated here provide the potential for more dexterous low-latency wireless motor prostheses. Whether ECoG BCIs can approach the communication performance demonstrated here remains to be determined.

\section{Leveraging Broadband Wireless Recording}

While the appropriate recording fidelity for stable, highperformance intracortical BCIs in people remains an active area of research, recent advances in high-performance iBCIs have leveraged broadband recording by decoding discrete spike rates combined with power in high-frequency local field potentials (HF-LFPs) up to $450 \mathrm{~Hz}$ [7], [12], [16] or $5 \mathrm{kHz}$ [4], [27]. This "hybrid" decoding has contributed to stable high performance iBCI cursor control [4], [7] and rapid decoder calibration [27]. This and other BBCI studies have incorporated common average referencing computed across a subset of electrodes at high sample rate (e.g., $500 \mathrm{~Hz}$ [16]; $15 \mathrm{kHz}$ [4], [7], [27]) to reduce noise prior to band pass filtering that could otherwise alias noise into the features computed for decoding. This technique computes a common signal from a subset of channels in realtime and applies it to all electrodes prior to computing neural features for decoding. Here, threshold crossing spike rates were computed after first applying a non-causal (bidirectional twopass) bandpass filter with a $5 \mathrm{kHz}$ upper cutoff frequency that has been shown to measurably improve decoding and BCI cursor performance relative to simple causal thresholding [29]. While we have not yet rigorously established a precise bandwidth that is necessary or sufficient for iBCI performance and stability for people with tetraplegia, these current practices provide a possible reference point for future optimized wireless BCIs.

\section{Future Work}

One advantage of recent ECoG or stent-based BCIs relative to the external wireless system in the current study is that they are fully implanted with no percutaneous connections. A fullyimplantable version of the BWD [25], [26], modified with a titanium package and inductive power, has been tested in animals and is being readied for regulatory testing prior to potential deployment in human clinical trials. To that end, the wireless device used in this study incorporates major elements of the fully implanted design and represents an externallymounted prototype of those core technologies. We have also demonstrated a prototype battery-powered mobile embedded decoding system [62]-[64] that integrates with both the external and fully implanted transmitters and is readily interfaced with other future electrode technologies with hundreds or thousands of contacts.

When integrated with stimulation capabilities, human wireless intracortical systems will offer new opportunities beyond iBCI [65] in neural sensing and closed-loop neuromodulation, including the treatment of seizures and neuropsychiatric disease [66]-[68]. Limitations of this current generation BWD and its implantable sibling are their unidirectional communication; neither stimulation nor impedance testing are currently possible. ASIC chips are being actively developed to introduce stimulation and impedance spectroscopy to future generations of this family of wireless devices.

Looking to the future, features of neural activity beyond spiking (e.g., high-frequency field potential oscillations, fieldfield phase coherence, spike-field relationships) remain to be fully explored in people and could one day be leveraged to enhance iBCI capabilities, performance and robustness. High resolution intracortical recording - currently only feasible as part of clinical research endeavors - will facilitate continued investigation of aspects of neural activity that may be clinically useful. We anticipate significant advances and paradigm shifts in neural signal processing, decoding algorithms, and control frameworks that will advance neural decoding for effective iBCI and neuromodulation applications. When, how and if different groups (academic, industry, etc.) should diverge in their efforts to develop better and clinically viable iBCI systems is a discussion central to iBCIs' successful translation. 


\section{CONCLUSION}

This study reports important progress in the clinical translation of an iBCI toward a future assistive medical device for individuals with paralysis. We demonstrate the first highresolution broadband recording from multiple implanted microelectrode arrays using a wireless $\mathrm{iBCI}$ in human subjects. Results with two individuals with tetraplegia demonstrate the viability of a wireless iBCI for real-time control of a point-andselect interface. Characteristics of the BWD recordings were highly comparable to those of wired recordings. This broadband wireless system also enables ongoing fundamental research into cortical processing during every day human behavior to inform future neuroscience and BCI advancements. This work overcomes former barriers to in-home mobile independent use of a promising assistive technology to restore communication and digital access for individuals with severe speech and/or motor impairments.

\section{ACKNOWLEDGMENT}

We thank participants T10, T5 and their families and caregivers; K. Barnabe, M. Bowker, B. Travers and Y. Mironovas.

Conflict of Interest: The MGH Translational Research Center has a clinical research support agreement with Neuralink Corp., Paradromics, and Synchron, for which LRH provides consultative input. KVS is a consultant to Neuralink Corp., and on the Scientific Advisory Boards of CTRL-Labs, Inscopix Inc., Heal Inc. and Mind X. JMH is a consultant for Neuralink Corp., Proteus Biomedical and Boston Scientific, and serves on the Medical Advisory Boards of Enspire DBS and Circuit Therapeutics.

Disclaimer: The content is solely the responsibility of the authors and does not necessarily represent the official views of the NIH, the Department of Veterans Affairs or the US Government.

Caution: Investigational Device. Limited by Federal Law to Investigational Use.

\section{REFERENCES}

[1] L. R. Hochberg et al., "Neuronal ensemble control of prosthetic devices by a human with tetraplegia," Nature, vol. 442, no. 7099, pp. 164-171, 2006.

[2] L. R. Hochberg et al., "Reach and grasp by people with tetraplegia using a neurally controlled robotic arm," Nature, vol. 485, no. 7398, pp. 372 5, May 2012.

[3] J. L. Collinger et al., "High-performance neuroprosthetic control by an individual with tetraplegia," Lancet, vol. 381, no. 9866, pp. 557-64, Feb. 2013.

[4] B. Jarosiewicz et al., "Virtual typing by people with tetraplegia using a self-calibrating intracortical brain-computer interface," Sci. Transl. Med., vol. 7, no. 313, p. 313ra179, 2015.

[5] T. Aflalo et al., "Decoding motor imagery from the posterior parietal cortex of a tetraplegic human," Science (80-. )., vol. 348, no. 6237, pp. 906-910, May 2015.

[6] C. E. Bouton et al., "Restoring cortical control of functional movement in a human with quadriplegia," Nature, vol. 533, no. 7602, pp. 247-250, Apr. 2016.

[7] C. Pandarinath et al., "High performance communication by people with paralysis using an intracortical brain-computer interface," Elife, vol. 6, p. e18554, Feb. 2017.

[8] P. Nuyujukian et al., "Cortical control of a tablet computer by people with paralysis," PLoS One, vol. 13, no. 11, p. e0204566, Nov. 2018.

[9] S. D. Stavisky et al., "Neural ensemble dynamics in dorsal motor cortex during speech in people with paralysis," Elife, vol. 8, Dec. 2019.

[10] S. D. Stavisky, F. R. Willett, D. T. Avansino, L. R. Hochberg, K. V. Shenoy, and J. M. Henderson, "Speech-related dorsal motor cortex activity does not interfere with iBCI cursor control," J Neural Eng, 2019. In press.

[11] B. Wodlinger, J. E. Downey, E. C. Tyler-Kabara, a B. Schwartz, M. L. Boninger, and J. L. Collinger, "Ten-dimensional anthropomorphic arm control in a human brain-machine interface: difficulties, solutions, and limitations," J. Neural Eng., vol. 12, no. 1, p. 016011, Feb. 2015.

[12] A. B. Ajiboye et al., "Restoration of reaching and grasping movements through brain-controlled muscle stimulation in a person with tetraplegia: a proof-of-concept demonstration," Lancet, vol. 389, no. 10081, pp. 1821-1830, Mar. 2017.

[13] D. Bacher et al., "Neural point-and-click communication by a person with incomplete locked-in syndrome," Neurorehabil. Neural Repair, vol. 29, no. 5, pp. 462-71, 2015.

[14] S.-P. P. Kim, J. D. Simeral, L. R. Hochberg, J. P. Donoghue, G. M. Friehs, and M. J. Black, "Point-and-click cursor control with an intracortical neural interface system by humans with tetraplegia," IEEE Trans Neural Syst Rehabil Eng, vol. 19, no. 2, pp. 193-203, Apr. 2011.

[15] J. D. Simeral, S. P. Kim, M. J. Black, J. P. Donoghue, and L. R. Hochberg, "Neural control of cursor trajectory and click by a human with tetraplegia 1000 days after implant of an intracortical microelectrode array," J Neural Eng, vol. 8, no. 2, p. 025027, Apr. 2011.

[16] V. Gilja et al., "Clinical translation of a high-performance neural prosthesis," Nat. Med., vol. 21, no. 10, pp. 1142-1145, Sep. 2015.

[17] J. A. Perge et al., "Intra-day signal instabilities affect decoding performance in an intracortical neural interface system," J. Neural Eng., vol. 10, no. 3, p. 036004, Jun. 2013.

[18] J. A. Perge et al., "Reliability of directional information in unsorted spikes and local field potentials recorded in human motor cortex," $J$. Neural Eng., vol. 11, no. 4, p. 046007, Aug. 2014.

[19] M. L. Homer, J. A. Perge, M. J. Black, M. T. Harrison, S. S. Cash, and L. R. Hochberg, "Adaptive offset correction for intracortical braincomputer interfaces," IEEE Trans. neural Syst. Rehabil. Eng., vol. 22, no. 2, pp. 239-48, Mar. 2014.

[20] B. Jarosiewicz et al., "Virtual typing by people with tetraplegia using a stabilized, self-calibrating intracortical brain-computer interface," in IEEE BRAIN Grand Challenges Conference, Washington, DC, 2014, vol. 7, no. 313, pp. 1-11.

[21] T. Milekovic et al., "Stable long-term BCI-enabled communication in ALS and locked-in syndrome using LFP signals," J. Neurophysiol., vol. 120, no. 1, pp. 343-360, Jul. 2018.

[22] N. Even-Chen et al., "Intracortical neural interface design opportunities for an order of magnitude power saving," Nat. Biomed. Eng., 2020. In press.

[23] M. Yin et al., "An externally head-mounted wireless neural recording device for laboratory animal research and possible human clinical use," in Proceedings of the Annual International Conference of the IEEE Engineering in Medicine and Biology Society, EMBS, 2013, pp. 3109 3114.

[24] M. Yin et al., "Wireless neurosensor for full-spectrum electrophysiology recordings during free behavior," Neuron, vol. 84, no. 6, pp. 1170-1182, Dec. 2014.

[25] D. A. Borton, M. Yin, J. Aceros, and A. Nurmikko, "An implantable wireless neural interface for recording cortical circuit dynamics in moving primates," J. Neural Eng., vol. 10, p. 26010, 2013.

[26] M. Yin, D. A. Borton, J. Aceros, W. R. Patterson, and A. V. Nurmikko, "A 100-channel hermetically sealed implantable device for chronic wireless neurosensing applications," IEEE Trans. Biomed. Circuits Syst., vol. 7, pp. 115-128, 2013.

[27] D. M. Brandman et al., "Rapid calibration of an intracortical braincomputer interface for people with tetraplegia," J. Neural Eng., vol. 15, no. 2, p. 026007 , Apr. 2018.

[28] J. Albites Sanabria, "BrainGate-enabled intracortical neural control of commercial tablet computers by individuals with tetraplegia," Brown University, 2016.

[29] N. Y. Masse et al., "Non-causal spike filtering improves decoding of movement intention for intracortical BCIs," J. Neurosci. Methods, Aug. 2014.

[30] P. Nuyujukian, J. M. Fan, J. C. Kao, S. I. Ryu, and K. V Shenoy, “A high-performance keyboard neural prosthesis enabled by task optimization," IEEE Trans. Biomed. Eng., pp. 1-9, Sep. 2015.

[31] P. Nuyujukian, J. C. Kao, J. M. Fan, S. D. Stavisky, S. I. Ryu, and K. V Shenoy, "Performance sustaining intracortical neural prostheses," $J$. 
Neural Eng., vol. 11, no. 6, p. 066003 , Dec. 2014.

[32] F. Wood, M. J. Black, C. Vargas-Irwin, M. Fellows, and J. P. Donoghue, "On the variability of manual spike sorting," IEEE Trans. Biomed. Eng., vol. 51, no. 6, pp. 912-8, Jun. 2004.

[33] C. Vargas-Irwin and J. P. Donoghue, "Automated spike sorting using density grid contour clustering and subtractive waveform decomposition," J. Neurosci. Methods, vol. 164, no. 1, pp. 1-18, Aug. 2007.

[34] S. Perel et al., "Single-unit activity, threshold crossings, and local field potentials in motor cortex differentially encode reach kinematics," $J$. Neurophysiol., vol. 114, no. 3, pp. 1500-1512, 2015.

[35] S. Todorova, P. Sadtler, A. Batista, S. Chase, and V. Ventura, "To sort or not to sort: the impact of spike-sorting on neural decoding performance.," J. Neural Eng., vol. 11, no. 5, p. 056005, Aug. 2014.

[36] B. P. Christie et al., "Comparison of spike sorting and thresholding of voltage waveforms for intracortical brain-machine interface performance," J. Neural Eng., vol. 12, no. 1, p. 016009, Mar. 2015.

[37] J. Dai et al., "Reliability of motor and sensory neural decoding by threshold crossings for intracortical brain-machine interface," J. Neural Eng., vol. 16, no. 3, p. 036011, Jun. 2019.

[38] G. W. Fraser, S. M. Chase, A. Whitford, and A. B. Schwartz, "Control of a brain-computer interface without spike sorting," J. Neural Eng., vol. 6, no. 5, p. 055004 , Oct. 2009.

[39] C. A. Chestek et al., "Long-term stability of neural prosthetic control signals from silicon cortical arrays in rhesus macaque motor cortex," $J$. Neural Eng., vol. 8, no. 4, p. 045005, Aug. 2011.

[40] E. M. Trautmann et al., "Accurate estimation of neural population dynamics without spike sorting," Neuron, vol. 103, no. 2, 2019.

[41] N. A. Steinmetz, C. Koch, K. D. Harris, and M. Carandini, "Challenges and opportunities for large-scale electrophysiology with Neuropixels probes," Curr. Opin. Neurobiol., vol. 50, pp. 92-100, Jun. 2018.

[42] M. M. Maharbiz, R. Muller, E. Alon, J. M. Rabaey, and J. M. Carmena, "Reliable next-generation cortical interfaces for chronic brain-machine interfaces and neuroscience," Proc. IEEE, vol. 105, no. 1, pp. 73-82, Jan. 2017.

[43] A. Obaid et al., "Massively parallel microwire arrays integrated with CMOS chips for neural recording," bioRxiv, p. 573295, Mar. 2019.

[44] E. Musk and Neuralink, "An integrated brain-machine interface platform with thousands of channels," J. Med. Internet Res., vol. 21, no. 10, p. e16194, Oct. 2019.

[45] R. M. Neely, D. K. Piech, S. R. Santacruz, M. M. Maharbiz, and J. M. Carmena, "Recent advances in neural dust: towards a neural interface platform.," Curr. Opin. Neurobiol., vol. 50, pp. 64-71, Jan. 2018.

[46] F. Laiwalla et al., "A distributed wireless network of implantable sub$\mathrm{mm}$ cortical microstimulators for brain-computer interfaces," in 2019 41 st Annual International Conference of the IEEE Engineering in Medicine and Biology Society (EMBC), 2019, pp. 6876-6879.

[47] J. A. Fernandez-Leon et al., "A wireless transmission neural interface system for unconstrained non-human primates," J. Neural Eng., vol. 12, no. 5, p. 056005 , Oct. 2015.

[48] C. A. Chestek et al., "HermesC: Low-power wireless neural recording system for freely moving primates," IEEE Trans. Neural Syst. Rehabil. Eng., vol. 17, pp. 330-338, 2009.

[49] D. A. Schwarz et al., "Chronic, wireless recordings of large-scale brain activity in freely moving rhesus monkeys," Nat. Methods, vol. 11, no. 6, pp. 670-6, Jun. 2014.

[50] H. Gao et al., "HermesE: A 96-channel full data rate direct neural interface in $0.13 \mu \mathrm{m}$ CMOS," IEEE J. Solid-State Circuits, vol. 47, no. 4, pp. 1043-1055, 2012.

[51] S. Rajangam et al., "Wireless cortical brain-machine interface for whole-body navigation in primates," Sci. Rep., vol. 6, p. 22170, Jan. 2016.

[52] J. D. Foster, O. Freifeld, P. Nuyujukian, S. I. Ryu, M. J. Black, and K. V. Shenoy, "Combining wireless neural recording and video capture for the analysis of natural gait," in 2011 5th International IEEE/EMBS Conference on Neural Engineering, 2011, vol. 2011, pp. 613-616.

[53] J. D Foster et al., "A freely-moving monkey treadmill model," J. Neural Eng., vol. 11, no. 4, p. 046020 , Aug. 2014.

[54] P. R. Kennedy and R. A. Bakay, "Restoration of neural output from a paralyzed patient by a direct brain connection," Neuroreport, vol. 9, no. 8, pp. 1707-1711, 1998.

[55] W. Wang et al., "An electrocorticographic brain interface in an individual with tetraplegia," PLoS One, vol. 8, no. 2, p. e55344, Jan. 2013.

[56] T. J. Oxley et al., "Minimally invasive endovascular stent-electrode array for high-fidelity, chronic recordings of cortical neural activity," Nat. Biotechnol., vol. 34, no. 3, pp. 320-327, Mar. 2016.

[57] S. Stanslaski et al., "A chronically implantable neural coprocessor for investigating the treatment of neurological disorders," IEEE Trans. Biomed. Circuits Syst., vol. 12, no. 6, pp. 1230-1245, Dec. 2018.

[58] M. J. Vansteensel et al., "Fully implanted brain-computer interface in a locked-in patient with ALS," N. Engl. J. Med., vol. 375, no. 21, pp. 2060-2066, Nov. 2016.

[59] N. C. Swann et al., "Chronic multisite brain recordings from a totally implantable bidirectional neural interface: experience in 5 patients with Parkinson's disease," J. Neurosurg., vol. 128, no. 2, pp. 605-616, Feb. 2018.

[60] K. Matsushita et al., "A fully implantable wireless ECoG 128-channel recording device for human brain-machine interfaces: W-HERBS," Front. Neurosci., vol. 12, p. 511, Jul. 2018.

[61] A. L. Benabid et al., "An exoskeleton controlled by an epidural wireless brain-machine interface in a tetraplegic patient: a proof-of-concept demonstration," Lancet Neurol., vol. 18, no. 12, pp. 1112-1122, Dec. 2019.

[62] J. Komar, C. Heelan, J. D. Simeral, and A. V Nurmikko, "A programmable 192-channel wireless neural signal processing and decoding platform for wearable mobile applications," in IEEE BRAIN Grand Challenges Conference, Washington, DC, 2014.

[63] C. Heelan, J. Komar, C. Vargas-Irwin, J. Simeral, and A. Nurmikko, "A mobile embedded platform for high performance neural signal computation and communication," in IEEE Biomedical Circuits and Systems Conference (BioCAS). Program 1316., 2015.

[64] C. Heelan, J. Komar, A. Nurmikko, and J. Simeral, "A mobile highperformance intracortical BCI with integrated antenna-receiver," Progr. No. 230.06. 2017 Neurosci. Meet. Planner. Washington, DC Soc. Neurosci. Online., 2017.

[65] S. N. Flesher et al., "Intracortical microstimulation of human somatosensory cortex," Sci. Transl. Med., vol. 8, no. 361, pp. 361ra141361ra141, Oct. 2016

[66] M. Pais-Vieira et al., "A closed loop brain-machine interface for epilepsy control using dorsal column electrical stimulation," Sci. Rep., vol. 6, no. 1, p. 32814, Dec. 2016.

[67] Y. S. Park et al., "Early detection of human epileptic seizures based on intracortical microelectrode array signals," IEEE Trans. Biomed. Eng., pp. 1-1, 2019.

[68] E. Greenwald, M. R. Masters, and N. V Thakor, "Implantable neurotechnologies: bidirectional neural interfaces--applications and VLSI circuit implementations," Med. Biol. Eng. Comput., vol. 54, no. 1, pp. 1-17, Jan. 2016. 